\title{
Short Term Effect and Safety of Antidiuretic Hormone in the Patients with Nocturia
}

\author{
Dong Il Kang ${ }^{1}$, Hak Min Kim ${ }^{1}$, Seung Yeob Oh ${ }^{1}$, Jang Ho Yoon ${ }^{1}$, Hyun Min Kim² ${ }^{2}$ Kweon Sik Min ${ }^{1,3}$ \\ ${ }^{1}$ Department of Urology, Pusan Paik Hospital, Inje University College of Medicine, Busan; \\ ${ }^{2}$ Department of Urology, Sahmyoook Medical Center, Seoul; \\ ${ }^{3}$ Paik Institute of Clinical Research, Pusan Paik Hospital, Inje University College of Medicine, Busan, Korea
}

\begin{abstract}
Purpose: To investigate the short-term safety of antidiuretic hormone in elderly patients with nocturnal polyuria, focus on hyponatremia and others electrolytes disturbances and to assess short-term effects on nocturnal urine output and number of nocturnal voids.

Methods: Between June 2005 and August 2006, a total of 34 patients with nocturnal polyuria were orally administered $0.2 \mathrm{mg}$ desmopressin tablet at bedtime for two weeks. Serum sodium, others electrolytes, urine sodium and urine osmolarity were assessed in the third days, one week and two weeks after treatment with desmopressin and compared adult group ( $<65$ years of age) with elderly group ( $\geq 65$ years of age). We assessed the effect of desmopressin using a frequency-volume charts and analysed.

Results: In total 34 patients (20 adult, 14 elderly) were analyzed. Desmopressin treatment did not significantly change serum and urine electrolytes include soduim concentration in elderly patients comparied with adult patients. Serum sodium concentration below normal range was recorded in 2 patients in elderly group, but no serious adverse events occurred and recovered without sequelae. The mean number of nocturnal voids decresed (54\% reduction) and nocturnal urine output decreased (57\% reduction) after using desmopressin.

Conclusions: Desmopressin was well tolerated and effective in elderly patients with nocturnal polyuria without clinically significant hyponatremia.
\end{abstract}

Keywords: Desmopressin; Nocturia; Elderly; Hyponatremia

\section{INTRODUCTION}

Nocturia is a common cause of sleep disturbance in elderly people, and several studies have reported that its frequency increases as the patient ages [1-3]. It had generally been accepted that bladder and prostate diseases induce adult nocturia, but recently, the excessive production of urine has been found to be the major cause of nocturia. Various factors such as nocturnal polyuria, nocturnal detrusor overactivity, reduction of functional bladder capacity, and also abnormalities of the lower urinary tract have also been found to function as single or combined etiologic factors [4-6].

Normally, the secretion of antidiuretic hormone increases during the night and nocturnal urine output is reduced to minimize urine-induced awakening. A reduction of antidiuretic hormone secretion seems the major factor in the nocturia caused by nocturnal polyuria $[7,8]$. With aging, the secretion of antidiuretic hormone during the night declines to maintain a similar level during the day and night, and this lack of difference becomes the major cause of adult nocturia [8].

Changes in the level of antidiuretic hormone are the theoretical basis for the usage of desmopressin, which is an analogue of
Corresponding author: Kweon Sik Min

Department of Urology and Paik Institute of Clincal Research,

Pusan Paik Hospital, Inje University College of Medicine, 633-165

Gaegeum-dong, Busanjin-gu, Busan 614-735, Korea

TEL: +82-51-890-6932 / FAX: +82-51-892-9887

E-mail: kweonsikmin@medimail.co.kr

Submitted: November 8, 2010 / Accepted after revision: December 21, 2010
This is an Open Access article distributed under the terms of the Creative Commons Attribution Non-Commercial License (http://creativecommons.org/licenses/by-nc/3.0/) which permits unrestricted non-commercial use, distribution, and reproduction in any medium, provided the original work is properly cited. 
arginine vasopressin, an antidiuretic hormone secreted from the posterior lobe of the hypophysis. An incresing number of reports indicate that administration of desmopressin to patients with nocturia seems to improve their symptoms [9]. However, several studies have also reported that headache, vomiting, dizziness, heart failure, and hyponatremia often appear [10]. Hyponatremia in particular not only seems to occur more often in the elderly, but can also be fatal in this population; therefore, special caution is needed. Awareness of nocturia caused by nocturnal polyuria has been increasing in Korea, and therefore, the clinical use of desmopressin in adult nocturia patients is increasing. Only a few studies, however, have reported on the effects and safety of its administration. Therefore, although its effects are recognized, we are still fearful of the possible side effects.

Accordingly, we aimed to study the effects and also the incidence of adverse effects with desmopressin treatment.

\section{MATERIALS AND METHODS}

From June 2005 to August 2006, voiding diary information was collected from outpatients who visited the urology department in our hospital with complaints of nocturia. Among these patients, 34 adults, aged 29-71 years, experiencing nighttime urination of more than 2 times per night and also more than 33\% of urine volume/day, were enrolled.

At the first visit, information on the patient's medical history and voiding diary was collected, and physical examinations with liver and renal function tests were performed. Serum electrolytes, urine electrolytes, and urine osmolality were also measured to exclude patients showing abnormal antidiuretic hormone levels as the result of chlorpropamide, clofibrate, or carbamazepine medication or those with heart failure, renal failure, or urinary tract infection.

Drug safety was analyzed by comparing the results of serum electrolytes, renal function tests, urine electrolytes, and changes in urine osmolality on the 3rd, 7th, and 14th days after the start of medication. Other health problems compared with the previous point of administration were also investigated at the outpatient clinic by use of simple questionnaires and phone calls, and the treatment effect on nocturia was evaluated by comparing the data from each voiding diary on the number of urinations and the amount of urine recorded during the 3 days before the start of treatment and the 2 weeks of administration.

Antidiuretic hormone $(0.2 \mathrm{mg})$ was prescribed to the subjects to be administrated orally once a day, before bedtime. We gave directions to the subjects about limiting excessive water drinking after dinner and also their alcohol and caffeine intake during the medication period. We also provided information that side effects including headache, vomiting, dizziness, edema, hyponatremia, and seizures could occur during the study. The subjects were divided into the elderly group (older than 65 years old) and the younger adult group to compare changes in serum and urine electrolytes, before and after treatment, to investigate the occurrence of adverse events, and finally to define the efficacy of the treatment.

For the statistical analysis, we used SPSS (SPSS Inc., Chicago, IL, USA) and the paired t-test to verify the significance of the change in electrolyte levels, before and after the antidiuretic hormone medication, and $\mathrm{P}$-values less than 0.05 were considered to indicate statistical significance. Serum sodium levels between 135 and $145 \mathrm{mmol} / \mathrm{L}$ were considered normal, and levels under $135 \mathrm{mmol} / \mathrm{L}$ were defined as hyponatremia.

\section{RESULTS}

A total of 34 patients were included in this study, with 14 patients (mean age, $69 \pm 3.6$ years) included in the elderly group, and 20 patients (mean age, $54.3 \pm 8.09$ years) in the younger adult group. Comparing the results between the groups, average daily total urine volumes were $1,753 \pm 453 \mathrm{~mL}$ and $1,582 \pm 416 \mathrm{~mL}$, amounts of nighttime urine volume were $573.1 \pm 72.7 \mathrm{~mL}$ and $590 \pm 83.1$ $\mathrm{mL}$, night urination frequencies were $5.0 \pm 1.9$ and $5.0 \pm 2.0$ times, and average sleep time was $7.3 \pm 1.12$ and $7.6 \pm 1.16$ hours, respectively (Table 1).

The average serum sodium level of the total subjects changed from $141.0 \mathrm{mmol} / \mathrm{L}$ (baseline) to $139.6 \mathrm{mmol} / \mathrm{L}$ (3rd day), 140.3 $\mathrm{mmol} / \mathrm{L}$ (7th day), and $139.8 \mathrm{mmol} / \mathrm{L}$ (14th day), which was

Table 1. Baseline patient characteristics

\begin{tabular}{lcc}
\hline Characteristic & $\geq 65 \mathrm{yr}$ & $<65 \mathrm{yr}$ \\
\hline No. of patients & 14 & 20 \\
Age $(\mathrm{yr})$ & $69.0 \pm 3.6$ & $54.3 \pm 8.09$ \\
Body mass index $\left(\mathrm{kg} / \mathrm{m}^{2}\right)$ & $22.1 \pm 2.6$ & $23.4 \pm 4.0$ \\
Nocturnal volume $(\mathrm{mL})$ & $573.1 \pm 72.7$ & $590 \pm 83.1$ \\
Nocturnal volume weight $(\mathrm{mL} / \mathrm{kg})$ & $9.55 \pm 1.7$ & $9.27 \pm 1.62$ \\
24 hr urine volume $(\mathrm{mL})$ & $1,753 \pm 453$ & $1,582 \pm 416$ \\
No. of nocturnal void & $5.0 \pm 1.9$ & $5.0 \pm 2.0$ \\
Duration of night & $7.3 \pm 1.12$ & $7.6 \pm 1.16$ \\
\hline
\end{tabular}

Values are presented as mean \pm SD. 
Table 2. Values of significant variables before and after dosing with desmopressin compared to baselilne. There are no statistical significance in all compared to baseline

\begin{tabular}{|c|c|c|c|c|c|}
\hline & & Base & 3 days & 7 days & 14 days \\
\hline \multirow{5}{*}{$<65 \mathrm{yr}$} & Serum & & & & \\
\hline & $\mathrm{Na}(\mathrm{mmol} / \mathrm{L})$ & $140.79 \pm 2.65$ & $139.63 \pm 1.83$ & $140.05 \pm 2.29$ & $139.79 \pm 2.57$ \\
\hline & Urine & & & & \\
\hline & $\mathrm{Na}(\mathrm{mmol} / \mathrm{L})$ & $145.40 \pm 82.80$ & $147.75 \pm 53.41$ & $133.30 \pm 48.43$ & $131.40 \pm 47.09$ \\
\hline & Osmolarity (mOsm/kg) & $639.35 \pm 190.58$ & $680.65 \pm 209.53$ & $669.00 \pm 195.98$ & $712.50 \pm 156.58$ \\
\hline \multirow[t]{5}{*}{$\geq 65 \mathrm{yr}$} & Serum & & & & \\
\hline & $\mathrm{Na}(\mathrm{mmol} / \mathrm{L})$ & $141.50 \pm 2.17$ & $139.79 \pm 3.44$ & $140.79 \pm 2.48$ & $140.00 \pm 2.77$ \\
\hline & Urine & & & & \\
\hline & $\mathrm{Na}(\mathrm{mmol} / \mathrm{L})$ & $109.29 \pm 62.99$ & $104.21 \pm 51.39$ & $113.71 \pm 58.75$ & $120.57 \pm 69.21$ \\
\hline & Osmolarity (mOsm/kg) & $531.07 \pm 199.44$ & $671.86 \pm 180.99$ & $590.14 \pm 194.17$ & $636.93 \pm 164.76$ \\
\hline
\end{tabular}

Values are presented as mean $\pm \mathrm{SD}$.

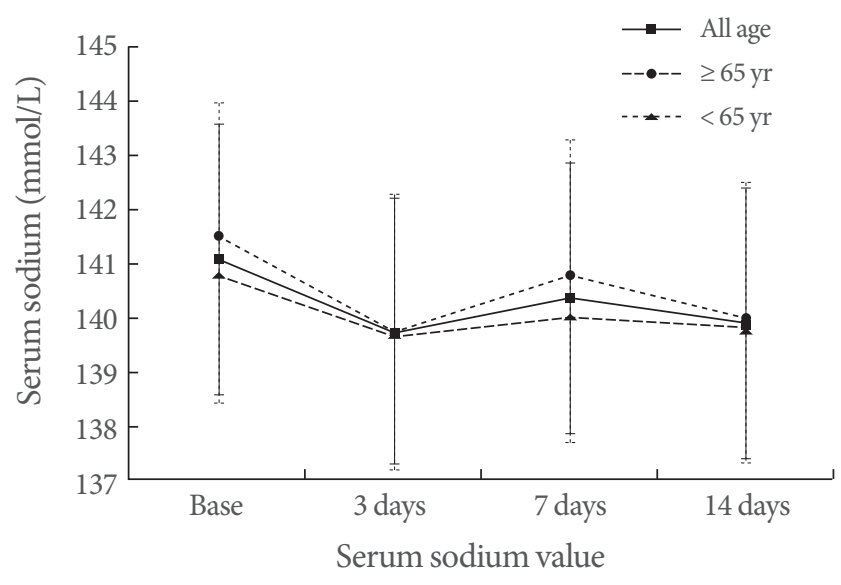

Fig. 1. The serum sodium values at base-line, the third, seventh and fourteenth days of desmopressin treatment. There are no statistical significance in all compared to baseline.

not a statically significant reduction after the desmopressin medication. Comparing levels measured on each day of both groups, the elderly group ( $\geq 65$ years old) showed a more reduction on the $3 \mathrm{rd}$ day $(141.5 \mathrm{mmol} / \mathrm{L}$ to $139.8 \mathrm{mmol} / \mathrm{L}$ to $140.8 \mathrm{mmol} / \mathrm{L}$ to $140 \mathrm{mmol} / \mathrm{L}$ at baseline and on days 3,7 , and 14 , respectively), and the younger adult group showed $140.7 \mathrm{mmol} / \mathrm{L}$ to 139.6 $\mathrm{mmol} / \mathrm{L}$ to $140.0 \mathrm{mmol} / \mathrm{L}$ to $139.7 \mathrm{mmol} / \mathrm{L}$ at baseline and on days 3,7 , and 14, respectively (Table 2, Fig. 1). However, these two groups did not show statistical significances.

Of the total 34 subjects, only 2 patients in the elderly group $(n=14)$ showed findings of hyponatremia, and their serum sodium levels returned to normal without any special treatments after stop of desmopressin. We also found no severe side effects such as clinically significant headache, nausea, abdominal pain,
Table 3. Number of patients with hyponatremia based on age and basal serum sodium

\begin{tabular}{llccc}
\hline Age & $\begin{array}{l}\text { Basal serum } \\
\text { sodium }(\mathrm{n})\end{array}$ & 3rd days & 7th days & 14th days \\
\hline$<65$ & Normal (20) & 0 & 0 & 0 \\
& Low (0) & 0 & 0 & 0 \\
$\geq 65$ & Normal (14) & 0 & 0 & 0 \\
& Low (0) & 2 & 0 & 0 \\
\hline
\end{tabular}

Table 4. The changes from baseline in voiding variables for all patients and by age

\begin{tabular}{lccc}
\hline Variable & All & $\geq 65 \mathrm{yr}$ & $<65 \mathrm{yr}$ \\
\hline No. of nocturnal voids & $-2.7 \pm 1.34$ & $-2.5 \pm 1.5$ & $-2.8 \pm 1.3$ \\
$\begin{array}{l}\text { Nocturnal urine } \\
\text { volume }(\mathrm{mL})\end{array}$ & $-288 \pm 122$ & $-260 \pm 131$ & $-309 \pm 116$ \\
\hline
\end{tabular}

Values are presented as mean \pm SD. $\mathrm{P}<0.05$ compared to baseline.

allergic reactions, or edema (Table 3). At the 3rd day, the urinary sodium level in the elderly group significantly decreased to $98 \%$ of the level before treatment, whereas the urine osmolality increased to $125 \%$, but without statistical significance compared to baseline. We also found no significant changes in blood or urine potassium, chloride, or creatinine levels.

Two weeks after the initiation of treatment, effects of the medication were seen in the decreased number of nocturia episode of $2.5 \pm 1.5$ in the elderly group and $2.8 \pm 1.3$ in the adult group. The amount of nocturia also decreased to $260 \pm 131 \mathrm{~mL}$ and $309 \pm 116 \mathrm{~mL}$ in each group, respectively, which were statistically significant improvements (Table 4). 


\section{DISCUSSION}

Nocturia is a major cause of decline in the quality of life of elderly people, and interest in this problem has been growing as a result of the increased proportion of the elderly population owing to their increasing life expectancy. Regardless of the cause, nocturia is a very common lower urinary tract symptom, and more than $72 \%$ of older people say that they have experienced nocturia more than at least once during their sleep, and $24 \%$ have experienced nocturia of three or more times per night $[11,12]$.

Because lower urinary tract abnormalities of the bladder or prostate are considered to be major causes of lower urinary tract symptoms, medications such as alpha blockers, 5-alpha reductase inhibitors, and anticholinergics or surgical treatments are usually considered as the first line of treatment. However, such treatments often result in no significant improvements.

Nocturia in elderly people can be classified into nocturnal polyuria, detrusor overactivity, mixed-type, and polyuria according to the cause, and nocturnal polyuria and detrusor overactivity account for more than $75 \%$ of cases [13]. Nocturnal polyuria is known to be related to changes in biological rhythms, over-intake of water, loss of body fluid, reduced concentrating ability of the proximal tubule induced by diuretics, change of in the glomerular filtration rate, insensitive diurnal variation of antidiuretic hormone, reduced secretion of renin-angiotensinaldosterone system, and also the secretion of atrial natriuretic peptide, caused by aging [14-16]. Detrusor overactivity could be induced by detrusor hyperreflexia caused by neurological diseases, urethral stricture, prostatic hyperplasia, and detrusor instability caused by bladder disorders; regardless of its cause, detrusor overactivity can induce nocturia [13]. Bladder outlet obstruction, detrusor overactivity, and the decrease in functional bladder capacity caused by neurogenic bladder disorder can also cause nocturia $[17,18]$.

Desmopressin, an analogue of the Antidiuretic hormone, arginine vasopressin, functions at the collecting duct to stimulate the reabsorption of water and reduce the production of urine. Desmopressin is a selective drug for central type diabetes insipidus with proven reliability. It is also known to have effects on adult nocturia caused by nocturnal polyuria [19]. However, it can potentially cause fatal side effects such as hyponatremia, caused by water retention. It has been reported in several studies that 12 to $22 \%$ of patients who are taking antidiuretic hormone analogues have experienced hyponatremia more than once. Another recent meta-analysis study of the risk of hypona- tremia in patients using this drug reported that hyponatremia accompanying clinical symptoms such as headache, nausea, vomiting, fatigue, dizziness, ataxia, or weight gain had occurred in 31 of the total 632 patients (4.9\%) [20-22]. The age of the patient was reported to be the most associated risk factor for hyponatremia; others included a serum sodium level lower than base line, urine output during the daytime higher than weight, and also increased body weight [22]. In addition to hyponatremia, desmopressin could also affect the excretion of electrolytes because urine osmolality increases after the administration of an antidiuretic hormone analogue, with increased urinary calcium excretion or decreased urinary potassium excretion of nocturia [23].

Antidiuretic hormone analogues have been used in the treatment of pediatric enuresis without any reports of serious side effects, and many reports on the administration of these drugs to adult nocturia patients have been published with no reports of severe side effects such as altered mental state, seizures, or congestive heart disease caused by water retention resulting in hyponatremia. Other mild clinical symptoms such as edema, nausea, and headache occurred only temporally or were resolved by discontinuing the medication or by simple treatments such as drinking sufficient water.

In this study, we also found that antidiuretic hormone analogue treatment of adults with nocturia significantly reduced nocturia and also the amount of urine, and we found no significant decrease in the serum sodium level. Of the total 34 subjects, only 2 patients in the elderly group showed symptoms of hyponatremia, but their levels returned to normal without any special treatments. We also found no clinically significant side effects such as headache, nausea, abdominal pain, allergic reactions, or edema.

In this short-term study of treatment of adult nocturia with antidiuretic hormone analogues, we observed no clinically significant adverse events caused by hyponatremia. It appears that this therapy could be safely used to treat adult nocturia patients. However, in elderly patients, sufficient guidelines about adequate water-drinking after dinner, limiting alcohol and caffeine intake, and routine body weight measurements should be provided, and follow-up studies to measure serum electrolytes should also be performed. Moreover, further studies about the longterm efficacy and safety of antidiuretic hormone analogue administration in adult nocturia patients should be continuously performed. 


\section{CONFLICT OF INTEREST}

No potential conflict of interest relevant to this article was reported.

\section{REFERENCES}

1. Chute CG, Panser LA, Girman CJ, Oesterling JE, Guess HA, Jacobsen SJ, et al. The prevalence of prostatism: a population-based survey of urinary symptoms. J Urol 1993;150:85-9.

2. Homma Y, Imajo C, Takahashi S, Kawabe K, Aso Y. Urinary symptoms and urodynamics in a normal elderly population. Scand J Urol Nephrol Suppl 1994;157:27-30.

3. Imai K, Okabe K, Kobayashi D, Ito K, Takahashi O, Yamanaka H, et al. Voiding disturbance in elderly males examined by prostate mass screening. Gunma Urological Oncology Study Group. Nippon Hinyokika Gakkai Zasshi 1991;82:1790-9.

4. Hunsballe JM, Rittig S, Pedersen EB, Olesen OV, Djurhuus JC. Single dose imipramine reduces nocturnal urine output in patients with nocturnal enuresis and nocturnal polyuria. J Urol 1997;158(3 Pt 1):830-6.

5. Simonsen O, Moller-Madsen B, Dorflinger T, Norgaard JP, Jorgensen HS, Lundhus E. The significance of age on symptoms and urodynamic- and cystoscopic findings in benign prostatic hypertrophy. Urol Res 1987;15:355-8.

6. Weiss JP, Blaivas JG. Nocturia. J Urol 2000;163:5-12.

7. Miller M. Nocturnal polyuria in older people: pathophysiology and clinical implications. J Am Geriatr Soc 2000;48:1321-9.

8. Asplund R. The nocturnal polyuria syndrome (NPS). Gen Pharmacol 1995;26:1203-9.

9. Mattiasson A, Abrams P, Van Kerrebroeck P, Walter S, Weiss J. Efficacy of desmopressin in the treatment of nocturia: a double-blind placebo-controlled study in men. BJU Int 2002;89:855-62.

10. Weatherall M. The risk of hyponatremia in older adults using des- mopressin for nocturia: a systematic review and meta-analysis. Neurourol Urodyn 2004;23:302-5.

11. Barker JC, Mitteness LS. Nocturia in the elderly. Gerontologist 1988;28:99-104.

12. Sommer P, Nielsen KK, Bauer T, Kristensen ES, Hermann GG, Steven $\mathrm{K}$, et al. Voiding patterns in men evaluated by a questionnaire survey. Br J Urol 1990;65:155-60.

13. Kim ET, Lee SI, Lee KS. The etiology and classification of nocturia in adults. Korean J Urol 2001;42:1075-79.

14. Asplund R. Nocturia in relation to sleep, health, and medical treatment in the elderly. BJU Int 2005;96 Suppl 1:15-21.

15. Chang SC, Lin AT, Chen KK, Chang LS. Multifactorial nature of male nocturia. Urology 2006;67:541-4.

16. Miller M. Nocturnal polyuria in older people: pathophysiology and clinical implications. J Am Geriatr Soc 2000;48:1321-9.

17. Weiss JP, Blaivas JG, Stember DS, Chaikin DC. Evaluation of the etiology of nocturia in men: the nocturia and nocturnal bladder capacity indices. Neurourol Urodyn 1999;18:559-65.

18. Weiss JP, Blaivas JG, Stember DS, Brooks MM. Nocturia in adults: etiology and classification. Neurourol Urodyn 1998;17:467-72.

19. Asplund R. Nocturia, nocturnal polyuria, and sleep quality in the elderly. J Psychosom Res 2004;56:517-25.

20. Mattiasson A, Abrams P, Van Kerrebroeck P, Walter S, Weiss J. Efficacy of desmopressin in the treatment of nocturia: a double-blind placebo-controlled study in men. BJU Int 2002;89:855-62.

21. Rembratt A, Norgaard JP, Andersson KE. Desmopressin in elderly patients with nocturia: short-term safety and effects on urine output, sleep and voiding patterns. BJU Int 2003;91:642-6.

22. Rembratt A, Riis A, Norgaard JP. Desmopressin treatment in nocturia; an analysis of risk factors for hyponatremia. Neurourol Urodyn 2006;25:105-9.

23. Chang YL, Lin AT, Chen KK. Short-term effects of desmopressin on water and electrolyte excretion in adults with nocturnal polyuria. J Urol 2007;177:2227-9. 